\title{
Parsing with Soft and Hard Constraints on Dependency Length*
}

\author{
Jason Eisner and Noah A. Smith \\ Department of Computer Science / Center for Language and Speech Processing \\ Johns Hopkins University, Baltimore, MD 21218 USA \\ \{jason, nasmith\}@es.jhu.edu
}

\begin{abstract}
In lexicalized phrase-structure or dependency parses, a word's modifiers tend to fall near it in the string. We show that a crude way to use dependency length as a parsing feature can substantially improve parsing speed and accuracy in English and Chinese, with more mixed results on German. We then show similar improvements by imposing hard bounds on dependency length and (additionally) modeling the resulting sequence of parse fragments. This simple "vine grammar" formalism has only finite-state power, but a context-free parameterization with some extra parameters for stringing fragments together. We exhibit a linear-time chart parsing algorithm with a low grammar constant.
\end{abstract}

\section{Introduction}

Many modern parsers identify the head word of each constituent they find. This makes it possible to identify the word-to-word dependencies implicit in a parse. ${ }^{1}$ (Some parsers, known as dependency parsers, even return these dependencies as their primary output.)

Why bother to identify these dependencies? The typical reason is to model the fact that some word pairs are more likely than others to engage in a dependency relationship. ${ }^{2}$ In this paper, we propose a different reason to identify dependencies in candidate parses: to evaluate not the dependency's word pair but its length (i.e., the string distance between the two words). Dependency lengths differ from

* This work was supported by NSF ITR grant IIS-0313193 to the first author and a fellowship from the Fannie and John Hertz Foundation to the second author. The views expressed are not necessarily endorsed by the sponsors. The authors thank Mark Johnson, Eugene Charniak, Charles Schafer, Keith Hall, and John Hale for helpful discussion and Elliott Drábek and Markus Dreyer for insights on (respectively) Chinese and German parsing. They also thank an anonymous reviewer for suggesting the German experiments.

${ }^{1}$ In a phrase-structure parse, if phrase $X$ headed by word token $x$ is a subconstituent of phrase $Y$ headed by word token $y \neq x$, then $x$ is said to depend on $y$. In a more powerful compositional formalism like LTAG or CCG, dependencies can be extracted from the derivation tree.

${ }^{2}$ It has recently been questioned whether these "bilexical" features actually contribute much to parsing performance (Klein and Manning, 2003; Bikel, 2004), at least when one has only a million words of training. typical parsing features in that they cannot be determined from tree-local information. Though lengths are not usually considered, we will see that bilexical dynamic-programming parsing algorithms can easily consider them as they build the parse.

Soft constraints. Like any other feature of trees, dependency lengths can be explicitly used as features in a probability model that chooses among trees. Such a model will tend to disfavor long dependencies (at least of some kinds), as these are empirically rare. In the first part of the paper, we show that such features improve a simple baseline dependency parser.

Hard constraints. If the bias against long dependencies is strengthened into a hard constraint that absolutely prohibits long dependencies, then the parser turns into a partial parser with only finite-state power. In the second part of the paper, we show how to perform chart parsing in asymptotic linear time with a low grammar constant. Such a partial parser does less work than a full parser in practice, and in many cases recovers a more precise set of dependencies (with little loss in recall).

\section{Short Dependencies in Langugage}

We assume that correct parses exhibit a "shortdependency preference": a word's dependents tend to be close to it in the string. ${ }^{3}$ If the $j^{\text {th }}$ word of a sentence depends on the $i^{\text {th }}$ word, then $|i-j|$ tends to be

\footnotetext{
${ }^{3}$ In this paper, we consider only a crude notion of "closeness": the number of intervening words. Other distance measures could be substituted or added (following the literature on heavy-shift and sentence comprehension), including the phonological, morphological, syntactic, or referential (given/new) complexity of the intervening material (Gibson, 1998). In parsing, the most relevant previous work is due to Collins (1997), who considered three binary features of the intervening material: did it contain (a) any word tokens at all, (b) any verbs, (c) any commas or colons? Note that (b) is effective because it measures the length of a dependency in terms of the number of alternative attachment sites that the dependent skipped over, a notion that could be generalized. Similarly, McDonald et al. (2005) separately considered each of the intervening POS tags.
} 
small. This implies that neither $i$ nor $j$ is modified by complex phrases that fall between $i$ and $j$. In terms of phrase structure, it implies that the phrases modifying word $i$ from a given side tend to be (1) few in number, (2) ordered so that the longer phrases fall farther from $i$, and (3) internally structured so that the bulk of each phrase falls on the side of $j$ away from $i$.

These principles can be blamed for several linguistic phenomena. (1) helps explain the "late closure" or "attach low" heuristic (e.g., Frazier, 1979; Hobbs and Bear, 1990): a modifier such as a PP is more likely to attach to the closest appropriate head. (2) helps account for heavy-shift: when an NP is long and complex, take NP out, put NP on the table, and give NP to Mary are likely to be rephrased as take out NP, put on the table NP, and give Mary $N P$. (3) explains certain non-canonical word orders: in English, a noun's left modifier must become a right modifier if and only if it is right-heavy (a taller politician vs. a politician taller than all her rivals ${ }^{4}$ ), and a verb's left modifier may extrapose its rightheavy portion (An aardvark walked in who had circumnavigated the globe ${ }^{5}$ ).

Why should sentences prefer short dependencies? Such sentences may be easier for humans to produce and comprehend. Each word can quickly "discharge its responsibilities," emitting or finding all its dependents soon after it is uttered or heard; then it can be dropped from working memory (Church, 1980; Gibson, 1998). Such sentences also succumb nicely to disambiguation heuristics that assume short dependencies, such as low attachment. Thus, to improve comprehensibility, a speaker can make stylistic choices that shorten dependencies (e.g., heavyshift), and a language can categorically prohibit some structures that lead to long dependencies ( $* a$ taller-than-all-her-rivals politician; *the sentence

\footnotetext{
${ }^{4}$ Whereas $* a$ politician taller and *a taller-than-all-herrivals politician are not allowed. The phenomenon is pervasive.

${ }^{5}$ This actually splits the heavy left dependent [an aardvark who ...] into two non-adjacent pieces, moving the heavy second piece. By slightly stretching the aardvark-who dependency in this way, it greatly shortens aardvark-walked. The same is possible for heavy, non-final right dependents: I met an aardvark yesterday who had circumnavigated the globe again stretches aardvark-who, which greatly shortens met-yesterday. These examples illustrate (3) and (2) respectively. However, the resulting non-contiguous constituents lead to non-projective parses that are beyond the scope of this paper.
}

that another sentence that had center-embedding was inside was incomprehensible).

Such functionalist pressures are not all-powerful. For example, many languages use SOV basic word order where SVO (or OVS) would give shorter dependencies. However, where the data exhibit some short-dependency preference, computer parsers as well as human parsers can obtain speed and accuracy benefits by exploiting that fact.

\section{Soft Constraints on Dependency Length}

We now enhance simple baseline probabilistic parsers for English, Chinese, and German so that they consider dependency lengths. We confine ourselves (throughout the paper) to parsing part-ofspeech (POS) tag sequences. This allows us to ignore data sparseness, out-of-vocabulary, smoothing, and pruning issues, but it means that our accuracy measures are not state-of-the-art. Our techniques could be straightforwardly adapted to (bi)lexicalized parsers on actual word sequences, though not necessarily with the same success.

\subsection{Grammar Formalism}

Throughout this paper we will use split bilexical grammars, or SBGs (Eisner, 2000), a notationally simpler variant of split head-automaton grammars, or SHAGs (Eisner and Satta, 1999). The formalism is context-free. We define here a probabilistic version, ${ }^{6}$ which we use for the baseline models in our experiments. They are only baselines because the SBG generative process does not take note of dependency length.

An SBG is an tuple $\mathcal{G}=(\Sigma, \$, L, R) . \quad \Sigma$ is an alphabet of words. (In our experiments, we parse only POS tag sequences, so $\Sigma$ is actually an alphabet of tags.) $\$ \notin \Sigma$ is a distinguished root symbol; let $\bar{\Sigma}=\Sigma \cup\{\$\}$. $L$ and $R$ are functions from $\bar{\Sigma}$ to probabilistic $\epsilon$-free finite-state automata over $\Sigma$. Thus, for each $w \in \bar{\Sigma}$, the SBG specifies "left" and "right" probabilistic FSAs, $L_{w}$ and $R_{w}$.

We use $\mathcal{L}_{w}(\mathcal{G}): \bar{\Sigma}^{*} \rightarrow[0,1]$ to denote the probabilistic context-free language of phrases headed by $w$. $\mathcal{L}_{w}(\mathcal{G})$ is defined by the following simple topdown stochastic process for sampling from it:

\footnotetext{
${ }^{6}$ There is a straightforward generalization to weighted SBGs, which need not have a stochastic generative model.
} 
1. Sample from the finite-state language $\mathcal{L}\left(L_{w}\right)$ a sequence $\lambda=w_{-1} w_{-2} \ldots w_{-\ell} \in \Sigma^{*}$ of left children, and from $\mathcal{L}\left(R_{w}\right)$ a sequence $\rho=$ $w_{1} w_{2} \ldots w_{r} \in \Sigma^{*}$ of right children. Each sequence is found by a random walk on its probabilistic FSA. We say the children depend on $w$.

2. For each $i$ from $-\ell$ to $r$ with $i \neq 0$, recursively sample $\alpha_{i} \in \Sigma^{*}$ from the context-free language $\mathcal{L}_{w_{i}}(\mathcal{G})$. It is this step that indirectly determines dependency lengths.

3. Return $\alpha_{-\ell} \ldots \alpha_{-2} \alpha_{-1} w \alpha_{1} \alpha_{2} \ldots \alpha_{r} \in \bar{\Sigma}^{*}$, a concatenation of strings.

Notice that $w$ 's left children $\lambda$ were generated in reverse order, so $w_{-1}$ and $w_{1}$ are its closest children while $w_{-\ell}$ and $w_{r}$ are the farthest.

Given an input sentence $\omega=w_{1} w_{2} \ldots w_{n} \in \Sigma^{*}$, a parser attempts to recover the highest-probability derivation by which $\$ \omega$ could have been generated from $\mathcal{L}_{\boldsymbol{\$}}(\mathcal{G})$. Thus, $\$$ plays the role of $w_{0}$. A sample derivation is shown in Fig. 1a. Typically, $L_{\$}$ and $R_{\$}$ are defined so that $\$$ must have no left children $(\ell=0)$ and at most one right child $(r \leq 1)$, the latter serving as the conventional root of the parse.

\subsection{Baseline Models}

In the experiments reported here, we defined only very simple automata for $L_{w}$ and $R_{w}(w \in \Sigma)$. However, we tried three automaton types, of varying quality, so as to evaluate the benefit of adding length-sensitivity at three different levels of baseline performance.

In model A (the worst), each automaton has topology $\odot \supset$, with a single state $q_{1}$, so token $w$ 's left dependents are conditionally independent of one another given $w$. In model $\mathrm{C}$ (the best), each automaton $\odot \longrightarrow \odot \supset$ has an extra state $q_{0}$ that allows the first (closest) dependent to be chosen differently from the rest. Model B is a compromise: ${ }^{7}$ it is like model A, but each type $w \in \Sigma$ may have an elevated or reduced probability of having no dependents at all. This is accomplished by using automata $\odot \longrightarrow \odot \supset$ as in model $\mathrm{C}$, which allows the stopping probabilities $p\left(\right.$ STOP $\left.\mid q_{0}\right)$ and $p$ (STOP $\left.\mid q_{1}\right)$ to differ, but tying the conditional dis-

\footnotetext{
${ }^{7}$ It is equivalent to the "dependency model with valence" of Klein and Manning (2004).
}

tributions $p\left(q_{0} \stackrel{w}{\longrightarrow} q_{1} \mid q_{0}, \neg \mathrm{STOP}\right)$ and $p\left(q_{1} \stackrel{w}{\longrightarrow} q_{1} \mid\right.$ $\left.q_{1}, \neg \mathrm{STOP}\right)$.

Finally, in $\S 3, L_{\$}$ and $R_{\$}$ are restricted as above, so $R_{\$}$ gives a probability distribution over $\Sigma$ only.

\subsection{Length-Sensitive Models}

None of the baseline models A-C explicitly model the distance between a head and child. We enhanced them by multiplying in some extra length-sensitive factors when computing a tree's probability. For each dependency, an extra factor $p(\Delta \mid \ldots)$ is multiplied in for the probability of the dependency's length $\Delta=|i-j|$, where $i$ and $j$ are the positions of the head and child in the surface string. ${ }^{8}$

Again we tried three variants. In one version, this new probability $p(\Delta \mid \ldots)$ is conditioned only on the direction $d=\operatorname{sign}(i-j)$ of the dependency. In another version, it is conditioned only on the POS $\operatorname{tag} h$ of the head. In a third version, it is conditioned on $d, h$, and the POS tag $c$ of the child.

\subsection{Parsing Algorithm}

Fig. 2a gives a variant of Eisner and Satta's (1999) SHAG parsing algorithm, adapted to SBGs, which are easier to understand. ${ }^{9}$ (We will modify this algorithm later in $\S 4$.) The algorithm obtains $O\left(n^{3}\right)$ runtime, despite the need to track the position of head words, by exploiting the conditional independence between a head's left children and right children. It builds "half-constituents" denoted by $\triangle$ (a head word together with some modifying phrases on the right, i.e., $w \alpha_{1} \ldots \alpha_{r}$ ) and $\Delta$ (a head word together with some modifying phrases on the left, i.e., $\left.\alpha_{-\ell} \ldots \alpha_{-1} w\right)$. A new dependency is introduced when $\triangle+\triangle$ are combined to get $\square$ or $\square$ (a pair of linked head words with all the intervening phrases, i.e., $w \alpha_{1} \ldots \alpha_{r} \alpha_{-\ell^{\prime}}^{\prime} \ldots \alpha_{-1}^{\prime} w^{\prime}$, where $w$ is respectively the parent or child of $w^{\prime}$ ). One can then combine $\square+\Delta=\Delta$, or

\footnotetext{
${ }^{8}$ Since the $\Delta$ values are fully determined by the tree but every $p(\Delta \mid \ldots) \leq 1$, this crude procedure simply reduces the probability mass of every legal tree. The resulting model is $d e-$ ficient (does not sum to 1); the remaining probability mass goes to impossible trees whose putative dependency lengths $\Delta$ are inconsistent with the tree structure. We intend in future work to explore non-deficient models (log-linear or generative), but even the present crude approach helps.

${ }^{9}$ The SHAG notation was designed to highlight the connection to non-split HAGs.
} 
$\Delta+\square=\Delta$. Only $O\left(n^{3}\right)$ combinations are possible in total when parsing a length- $n$ sentence.

\subsection{A Note on Word Senses}

[This section may be skipped by the casual reader.]

A remark is necessary about $: w$ and $: w^{\prime}$ in Fig. 2a, which represent senses of the words at positions $h$ and $h^{\prime}$. Like past algorithms for SBGs (Eisner, 2000), Fig. 2a is designed to be a bit more general and integrate sense disambiguation into parsing. It formally runs on an input $\Omega=W_{1} \ldots W_{n} \subseteq \Sigma^{*}$, where each $W_{i} \subseteq \Sigma$ is a "confusion set" over possible values of the $i^{\text {th }}$ word $w_{i}$. The algorithm recovers the highest-probability derivation that generates $\$ \omega$ for some $\omega \in \Omega$ (i.e., $\omega=w_{1} \ldots w_{n}$ with $\left.(\forall i) w_{i} \in W_{i}\right)$.

This extra level of generality is not needed for any of our experiments, but it is needed for SBG parsers to be as flexible as SHAG parsers. We include it in this paper to broaden the applicability of both Fig. 2a and our extension of it in $\S 4$.

The "senses" can be used in an SBG to pass a finite amount of information between the left and right children of a word, just as SHAGs allow. ${ }^{10}$ For example, to model the fronting of a direct object, an SBG might use a special sense of a verb, whose automata tend to generate both one more noun in $\lambda$ and one fewer noun in $\rho$.

Senses can also be used to pass information between parents and children. Important uses are to encode lexical senses, or to enrich the dependency parse with constituent labels or depen-

\footnotetext{
${ }^{10}$ Fig. 2a enhances the Eisner-Satta version with explicit senses while matching its asymptotic performance. On this point, see (Eisner and Satta, 1999, $\S 8$ and footnote 6). However, it does have a practical slowdown, in that START-LEFT nondeterministically guesses every possible sense of $W_{i}$, and these senses are pursued separately. To match the Eisner-Satta algorithm, we should not need to commit to a word's sense until we have seen all its left children. That is, left triangles and left trapezoids should not carry a sense $: w$ at all, except for the completed left triangle (marked F) that is produced by FINISHLEFT. FINISH-LEFT should choose a sense $w$ of $W_{h}$ according to the final state $q$, which reflects knowledge of $W_{h}$ 's left children. For this strategy to work, the transitions in $L_{w}$ (used by ATTACH-LEFT) must not depend on the particular sense $w$ but only on $W$. In other words, all $L_{w}: w \in W_{h}$ are really copies of a shared $L_{W_{h}}$, except that they may have different final states. This requirement involves no loss of generality, since the nondeterministic shared $L_{W_{h}}$ is free to branch as soon as it likes onto paths that commit to the various senses $w$.
}

dency labels (Eisner, 2000). For example, the input token $W_{i}=\left\{\right.$ bank $_{1} / N / N P$, bank $_{2} / N / N P$, bank $_{3} / V / V P$, bank $\left._{3} / V / S\right\} \subset \Sigma$ allows four "senses" of bank, namely two nominal meanings, and two syntactically different versions of the verbal meaning, whose automata require them to expand into VP and S phrases respectively.

The cubic runtime is proportional to the number of ways of instantiating the inference rules in Fig. 2a: $O\left(n^{2}\left(n+t^{\prime}\right) t g^{2}\right)$, where $n=|\Omega|$ is the input length, $g=\max _{i=1}^{n}\left|W_{i}\right|$ bounds the size of a confusion set, $t$ bounds the number of states per automaton, and $t^{\prime} \leq t$ bounds the number of automaton transitions from a state that emit the same word. For deterministic automata, $t^{\prime}=1 .^{11}$

\subsection{Probabilistic Parsing}

It is easy to make the algorithm of Fig. 2a lengthsensitive. When a new dependency is added by an ATTACH rule that combines $\triangle+\Delta$, the annotations on $\triangle$ and $\triangle$ suffice to determine the dependency's length $\Delta=\left|h-h^{\prime}\right|$, direction $d=\operatorname{sign}\left(h-h^{\prime}\right)$, head word $w$, and child word $w^{\prime} .{ }^{12}$ So the additional cost of such a dependency, e.g. $p\left(\Delta \mid d, w, w^{\prime}\right)$, can be included as the weight of an extra antecedent to the rule, and so included in the weight of the resulting $\square$ or $\square$.

To execute the inference rules in Fig. 2a, we use a prioritized agenda. Derived items such as $\triangle, \triangle, \square$, and $\square$ are prioritized by their Viterbi-inside probabilities. This is known as uniform-cost search or shortest-hyperpath search (Nederhof, 2003). We halt as soon as a full parse (the accept item) pops from the agenda, since uniform-cost search (as a special case of the $\mathrm{A}^{*}$ algorithm) guarantees this to be the maximumprobability parse. No other pruning is done.

\footnotetext{
${ }^{11}$ Confusion-set parsing may be regarded as parsing a particular lattice with $n$ states and $n g$ arcs. The algorithm can be generalized to lattice parsing, in which case it has runtime $O\left(m^{2}\left(n+t^{\prime}\right) t\right)$ for a lattice of $n$ states and $m$ arcs. Roughly, $h: w$ is replaced by an arc, while $i$ is replaced by a state and $i-1$ is replaced by the same state.

${ }^{12}$ For general lattice parsing, it is not possible to determine $\Delta$ while applying this rule. There $h$ and $h^{\prime}$ are arcs in the lattice, not integers, and different paths from $h$ to $h^{\prime}$ might cover different numbers of words. Thus, if one still wanted to measure dependency length in words (rather than in, say, milliseconds of speech), each item would have to record its width explicitly, leading in general to more items and increased runtime.
} 
With a prioritized agenda, a probability model that more sharply discriminates among parses will typically lead to a faster parser. (Low-probability constituents languish at the back of the agenda and are never pursued.) We will see that the lengthsensitive models do run faster for this reason.

\subsection{Experiments with Soft Constraints}

We trained models A-C, using unsmoothed maximum likelihood estimation, on three treebanks: the Penn (English) Treebank (split in the standard way, $\S 2-21$ train/ $\$ 23$ test, or $950 \mathrm{~K} / 57 \mathrm{~K}$ words), the Penn Chinese Treebank ( $80 \%$ train $/ 10 \%$ test or $508 \mathrm{~K} / 55 \mathrm{~K}$ words), and the German TIGER corpus $(80 \% / 10 \%$ or $539 \mathrm{~K} / 68 \mathrm{~K}$ words). ${ }^{13}$ Estimation was a simple matter of counting automaton events and normalizing counts into probabilities. For each model, we also trained the three length-sensitive versions described in $\S 3.3$.

The German corpus contains non-projective trees. None of our parsers can recover non-projective dependencies (nor can our models produce them). This fact was ignored when counting events for maximum likelihood estimation: in particular, we always trained $L_{w}$ and $R_{w}$ on the sequence of $w$ 's immediate children, even in non-projective trees.

Our results (Tab. 1) show that sharpening the probabilities with the most sophisticated distance factors $p(\Delta \mid d, h, c)$, consistently improved the speed of all parsers. ${ }^{14}$ The change to the code is trivial. The only overhead is the cost of looking up and multiplying in the extra distance factors.

Accuracy also improved over the baseline models of English and Chinese, as well as the simpler baseline models of German. Again, the most sophisticated distance factors helped most, but even the simplest distance factor usually obtained most of the accuracy benefit.

German model $\mathrm{C}$ fell slightly in accuracy. The speedup here suggests that the probabilities were sharpened, but often in favor of the wrong parses. We did not analyze the errors on German; it may

\footnotetext{
${ }^{13}$ Heads were extracted for English using Michael Collins' rules and Chinese using Fei Xia's rules (defaulting in both cases to right-most heads where the rules fail). German heads were extracted using the TIGER Java API; we discarded all resulting dependency structures that were cyclic or unconnected (6\%).

${ }^{14} \mathrm{We}$ measure speed abstractly by the number of items built and pushed on the agenda.
}

be relevant that $25 \%$ of the German sentences contained a non-projective dependency between nonpunctuation tokens.

Studying the parser output for English, we found that the length-sensitive models preferred closer attachments, with $19.7 \%$ of tags having a nearer parent in the best parse under model $\mathrm{C}$ with $p(\Delta \mid d, h, c)$ than in the original model $\mathrm{C}, 77.7 \%$ having a parent at the same distance, and only $2.5 \%$ having a farther parent. The surviving long dependencies (at any length $>1$ ) tended to be much more accurate, while the (now more numerous) length-1 dependencies were slightly less accurate than before.

We caution that length sensitivity's most dramatic improvements to accuracy were on the worse baseline models, which had more room to improve. The better baseline models (B and C) were already able to indirectly capture some preference for short dependencies, by learning that some parts of speech were unlikely to have multiple left or multiple right dependents. Enhancing $\mathrm{B}$ and $\mathrm{C}$ therefore contributed less, and indeed may have had some harmful effect by over-penalizing some structures that were already appropriately penalized. ${ }^{15}$ It remains to be seen, therefore, whether distance features would help state-of-the art parsers that are already much better than model C. Such parsers may already incorporate features that indirectly impose a good model of distance, though perhaps not as cheaply.

\section{Hard Dependency-Length Constraints}

We have seen how an explicit model of distance can improve the speed and accuracy of a simple probabilistic dependency parser. Another way to capitalize on the fact that most dependencies are local is to impose a hard constraint that simply forbids long dependencies.

The dependency trees that satisfy this constraint yield a regular string language. ${ }^{16}$ The constraint prevents arbitrarily deep center-embedding, as well as arbitrarily many direct dependents on a given head,

\footnotetext{
${ }^{15}$ Owing to our deficient model. A log-linear or discriminative model would be trained to correct for overlapping penalties and would avoid this risk. Non-deficient generative models are also possible to design, along lines similar to footnote 16 .

${ }^{16}$ One proof is to construct a strongly equivalent $\mathrm{CFG}$ without center-embedding (Nederhof, 2000). Each nonterminal has the form $\langle w, q, i, j\rangle$, where $w \in \Sigma, q$ is a state of $L_{w}$ or $R_{w}$, and $i, j \in\{0,1, \ldots k-1, \geq k\}$. We leave the details as an exercise.
} 


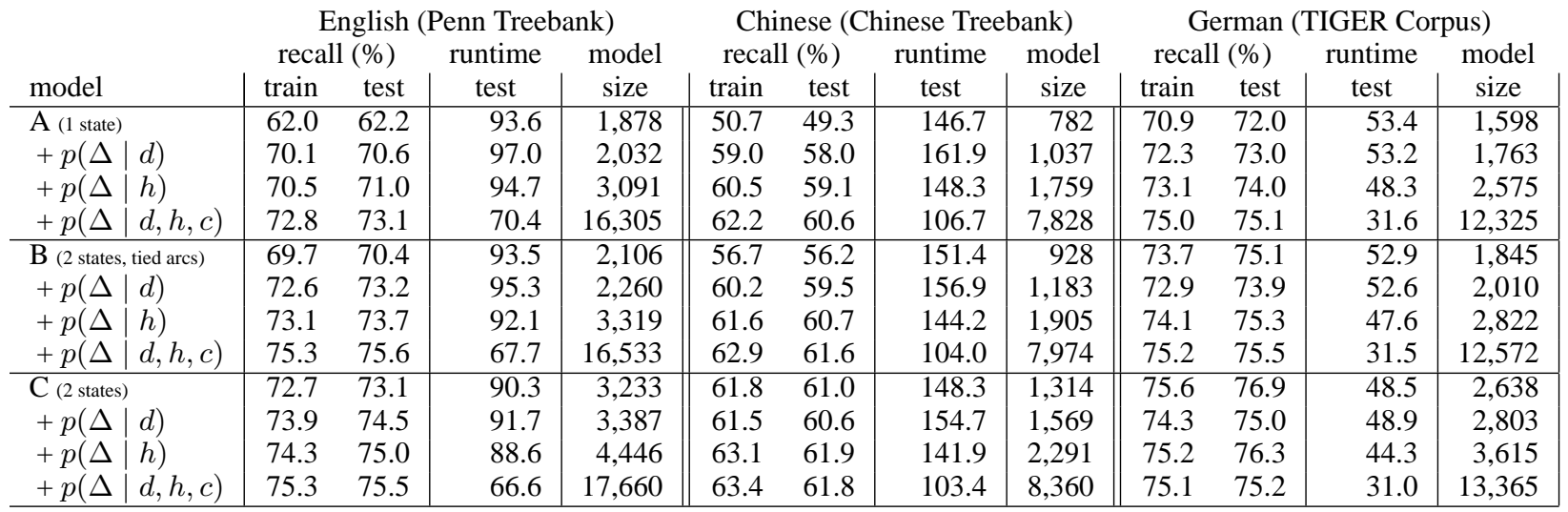

Table 1: Dependency parsing of POS tag sequences with simple probabilistic split bilexical grammars. The models differ only in how they weight the same candidate parse trees. Length-sensitive models are larger but can improve dependency accuracy and speed. (Recall is measured as the fraction of non-punctuation tags whose correct parent (if not the \$ symbol) was correctly recovered by the parser; it equals precision, unless the parser left some sentences unparsed (or incompletely parsed, as in $\S 4$ ), in which case precision is higher. Runtime is measured abstractly as the average number of items (i.e., $\square, \square, \square, \square$ ) built per word. Model size is measured as the number of nonzero parameters.)

either of which would allow the non-regular language $\left\{a^{n} b c^{n}: 0<n<\infty\right\}$. It does allow arbitrarily deep right- or left-branching structures.

\subsection{Vine Grammars}

The tighter the bound on dependency length, the fewer parse trees we allow and the faster we can find them using the algorithm of Fig. 2a. If the bound is too tight to allow the correct parse of some sentence, we would still like to allow an accurate partial parse: a sequence of accurate parse fragments (Hindle, 1990; Abney, 1991; Appelt et al., 1993; Chen, 1995; Grefenstette, 1996). Furthermore, we would like to use the fact that some fragment sequences are presumably more likely than others.

Our partial parses will look like the one in Fig. 1b. where 4 subtrees rather than 1 are dependent on $\$$. This is easy to arrange in the SBG formalism. We merely need to construct our SBG so that the automaton $R_{\$}$ is now permitted to generate multiple children-the roots of parse fragments.

This $R_{\$}$ is a probabilistic finite-state automaton that describes legal or likely root sequences in $\Sigma^{*}$. In our experiments in this section, we will train it to be a first-order (bigram) Markov model. (Thus we construct $R_{\$}$ in the usual way to have $|\Sigma|+1$ states, and train it on data like the other left and right automata. During generation, its state remembers the previously generated root, if any. Recall that we are working with POS tag sequences, so the roots, like all other words, are tags in $\Sigma$.)

The 4 subtrees in Fig. $1 \mathrm{~b}$ appear as so many bunches of grapes hanging off a vine. We refer to the dotted dependencies upon $\$$ as vine dependencies, and the remaining, bilexical dependencies as tree dependencies.

One might informally use the term "vine grammar" (VG) for any generative formalism, intended for partial parsing, in which a parse is a constrained sequence of trees that cover the sentence. In general, a VG might use a two-part generative process: first generate a finite-state sequence of roots, then expand the roots according to some more powerful formalism. Conveniently, however, SBGs and other dependency grammars can integrate these two steps into a single formalism.

\subsection{Feasible Parsing}

Now, for both speed and accuracy, we will restrict the trees that may hang from the vine. We define a feasible parse under our SBG to be one in which all tree dependencies are short, i.e., their length never exceeds some hard bound $k$. The vine dependencies may have unbounded length, of course, as in Fig. 1b.

Sentences with feasible parses form a regular language. This would also be true under other definitions of feasibility, e.g., we could have limited the depth or width of each tree on the vine. However, that would have ruled out deeply right-branching trees, which are very common in language, and 
would
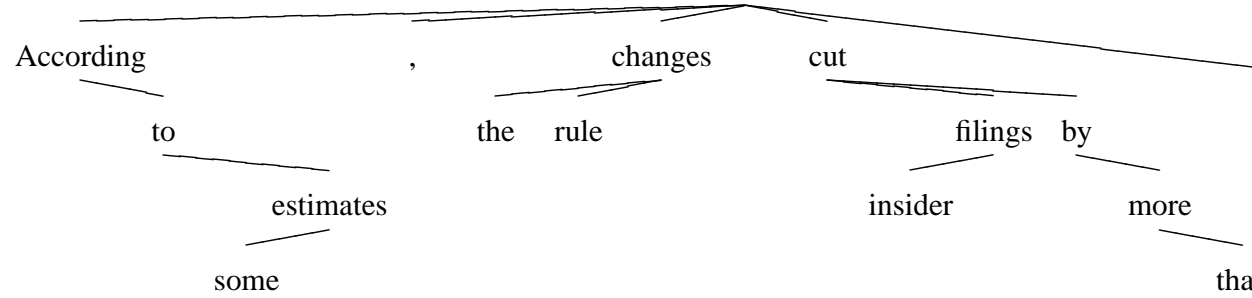

some

(b)

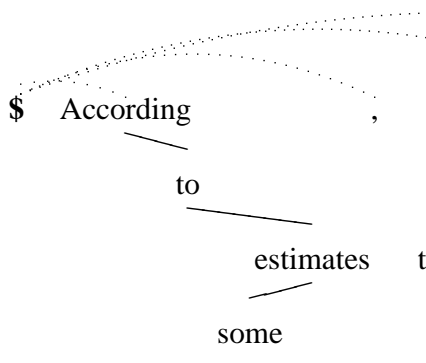

Figure 1: (a) A dependency tree on words. (Our experiments use only POS tags.) (b) A partial parse for the same sentence retaining only tree dependencies of length $\leq k=3$. The roots of the 4 resulting parse fragments are now connected only by their dotted-line "vine dependencies" on \$. Transforming (a) into (b) involves grafting subtrees rooted at "According”, ",", and "." onto the vine.

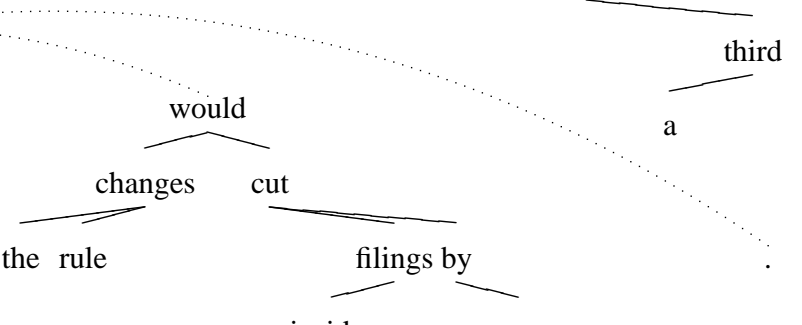

are also the traditional way to describe finite-state sublanguages within a context-free grammar. By contrast, our limitation on dependency length ensures regularity while still allowing (for any bound $k \geq 1$ ) arbitrarily wide and deep trees, such as $a \rightarrow b \rightarrow \ldots \rightarrow$ root $\leftarrow \ldots \leftarrow y \leftarrow z$.

Our goal is to find the best feasible parse (if any). Rather than transform the grammar as in footnote 16 , our strategy is to modify the parser so that it only considers feasible parses. The interesting problem is to achieve linear-time parsing with a grammar constant that is as small as for ordinary parsing.

We also correspondingly modify the training data so that we only train on feasible parses. That is, we break any long dependencies and thereby fragment each training parse (a single tree) into a vine of one or more restricted trees. When we break a childto-parent dependency, we reattach the child to $\$ .^{17}$ This process, grafting, is illustrated in Fig. 1. Although this new parse may score less than $100 \%$ recall of the original dependencies, it is the best feasible parse, so we would like to train the parser to find it. ${ }^{18}$ By training on the modified data, we learn more

\footnotetext{
${ }^{17}$ Any dependency covering the child must also be broken to preserve projectivity. This case arises later; see footnote 25 .

${ }^{18}$ Although the parser will still not be able to find it if it is non-projective (possible in German). Arguably we should have defined "feasible" to also require projectivity, but we did not.
}

appropriate statistics for both $R_{\$}$ and the other automata. If we trained on the original trees, we would inaptly learn that $R_{\$}$ always generates a single root rather than a certain kind of sequence of roots.

For evaluation, we score tree dependencies in our feasible parses against the tree dependencies in the unmodified gold standard parses, which are not necessarily feasible. We also show oracle performance.

\subsection{Approach \#1: FSA Parsing}

Since we are now dealing with a regular language, it is possible in principle to use a weighted finitestate automaton (FSA) to search for the best feasible parse. The idea is to find the highest-weighted path that accepts the input string $\omega=w_{1} w_{2} \ldots w_{n}$. Using the Viterbi algorithm, this takes time $O(n)$.

The trouble is that this linear runtime hides a constant factor, which depends on the size of the relevant part of the FSA and may be enormous for any correct FSA. ${ }^{19}$

Consider an example from Fig $1 \mathrm{~b}$. After nondeterministically reading $w_{1} \ldots w_{11}=$ According... insider along the correct path, the FSA state must record (at least) that insider has no parent yet and that $R_{\$}$ and $R_{c u t}$ are in particular states that

\footnotetext{
${ }^{19}$ The full runtime is $O(n E)$, where $E$ is the number of FSA edges, or for a tighter estimate, the number of FSA edges that can be traversed by reading $\omega$.
} 
may still accept more children. Else the FSA cannot know whether to accept a continuation $w_{12} \ldots w_{n}$.

In general, after parsing a prefix $w_{1} \ldots w_{j}$, the FSA state must somehow record information about all incompletely linked words in the past. It must record the sequence of past words $w_{i}(i \leq j)$ that still need a parent or child in the future; if $w_{i}$ still needs a child, it must also record the state of $R_{w_{i}}$.

Our restriction to dependency length $\leq k$ is what allows us to build a finite-state machine (as opposed to some kind of pushdown automaton with an unbounded number of configurations). We need only build the finitely many states where the incompletely linked words are limited to at most $w_{0}=\$$ and the $k$ most recent words, $w_{j-k+1} \ldots w_{j}$. Other states cannot extend into a feasible parse, and can be pruned.

However, this still allows the FSA to be in $O\left(2^{k} t^{k+1}\right)$ different states after reading $w_{1} \ldots w_{j}$. Then the runtime of the Viterbi algorithm, though linear in $n$, is exponential in $k$.

\subsection{Approach \#2: Ordinary Chart Parsing}

A much better idea for most purposes is to use a chart parser. This allows the usual dynamic programming techniques for reusing computation. (The FSA in the previous section failed to exploit many such opportunities: exponentially many states would have proceeded redundantly by building the same $w_{j+1} w_{j+2} w_{j+3}$ constituent.)

It is simple to restrict our algorithm of Fig. 2a to find only feasible parses. It is the ATTACH rules $\triangle+\triangle$ that add dependencies: simply use a side condition to block them from applying unless $\left|h-h^{\prime}\right| \leq k$ (short tree dependency) or $h=0$ (vine dependency). This ensures that all $\square$ and $\square$ will have width $\leq k$ or have their left edge at 0 .

One might now incorrectly expect runtime linear in $n$ : the number of possible ATTACH combinations is reduced from $O\left(n^{3}\right)$ to $O\left(n k^{2}\right)$, because $i$ and $h^{\prime}$ are now restricted to a narrow range given $h$.

Unfortunately, the half-constituents $\triangle$ and $\triangle$ may still be arbitrarily wide, thanks to arbitrary right- and left-branching: a feasible vine parse may be a sequence of wide trees $\triangle$. Thus there are $O\left(n^{2} k\right)$ possible COMPLETE combinations, not to mention $O\left(n^{2}\right)$ ATTACH-RIGHT combinations for which $h=0$. So the runtime remains quadratic.

\subsection{Approach \#3: Specialized Chart Parsing}

How, then, do we get linear runtime and a reasonable grammar constant? We give two ways to achieve runtime of $O\left(n k^{2}\right)$.

First, we observe without details that we can easily achieve this by starting instead with the algorithm of Eisner (2000), ${ }^{20}$ rather than Eisner and Satta (1999), and again refusing to add long tree dependencies. That algorithm effectively concatenates only trapezoids, not triangles. Each is spanned by a single dependency and so has width $\leq k$. The vine dependencies do lead to wide trapezoids, but these are constrained to start at 0 , where $\$$ is. So the algorithm tries at most $O\left(n k^{2}\right)$ combinations of the form ${ }_{h} \square_{i}+{ }_{i} \square_{j}$ (like the ATTACH combinations above) and $O(n k)$ combinations of the form ${ }_{0} \square_{i}+{ }_{i} \square_{j}$, where $i-h \leq k, j-i \leq k$. The precise runtime is $O\left(n k\left(k+t^{\prime}\right) t g^{3}\right)$.

We now propose a hybrid linear-time algorithm that further improves runtime to $O\left(n k\left(k+t^{\prime}\right) t g^{2}\right)$, saving a factor of $g$ in the grammar constant. ${ }^{21} \mathrm{We}$ observe that since within-tree dependencies must have length $\leq k$, they can all be captured within Eisner-Satta trapezoids of width $\leq k$. So our VG parse $\triangle *$ can be assembled by simply concatenating a sequence $(\triangle \square * \square * \bigsqcup$ )* of these narrow trapezoids interspersed with width- 0 triangles. As this is a regular sequence, we can assemble it in linear time from left to right (rather than in the order of Eisner and Satta (1999)), multiplying the items' probabilities together. Whenever we start adding the right half $\square * \triangle$ of a tree along the vine, we have discovered that tree's root, so we multiply in the probability of a $\$ \leftarrow$ root dependency.

Formally, our hybrid parsing algorithm restricts the original rules of Fig. 2a to build only trapezoids of width $\leq k$ and triangles of width $<k .^{22}$ The additional inference rules in Fig. $2 b$ then assemble the final VG parse as just described.

\footnotetext{
${ }^{20}$ With a small change that when two items are combined, the right item (rather than the left) must be simple.

${ }^{21}$ This savings comes from building the internal structure of a trapezoid from both ends inward rather than from left to right. The corresponding unrestricted algorithms (Eisner, 2000; Eisner and Satta, 1999, respectively) have exactly the same runtimes with $k$ replaced by $n$.

${ }^{22}$ For the experiments of $\S 4.7$, where $k$ varied by type, we restricted these rules as tightly as possible given $h$ and $h^{\prime}$.
} 


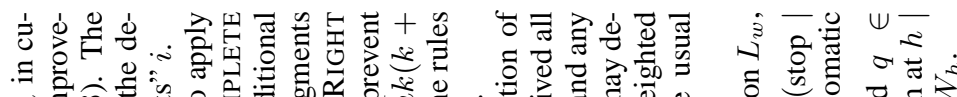

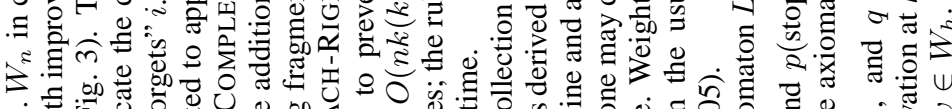

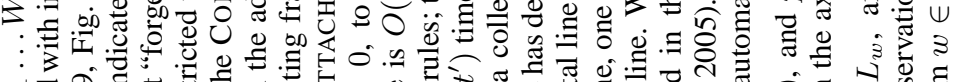

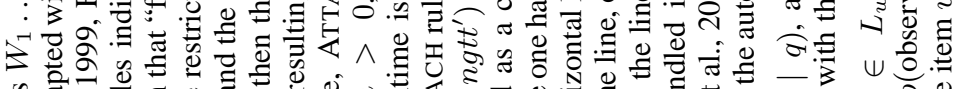

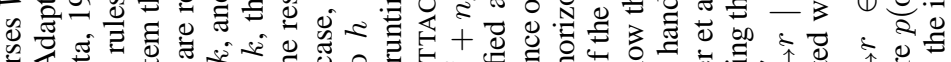

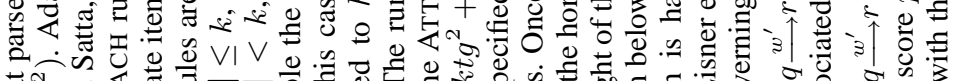

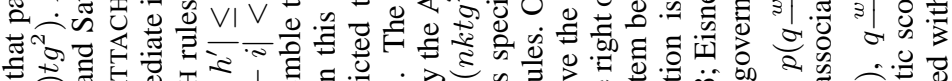

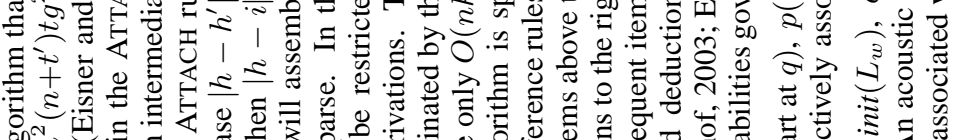

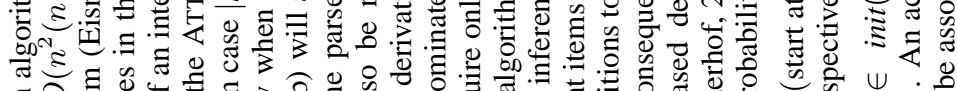

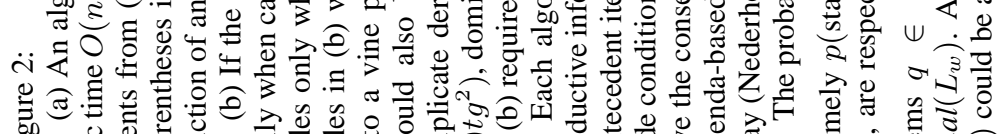

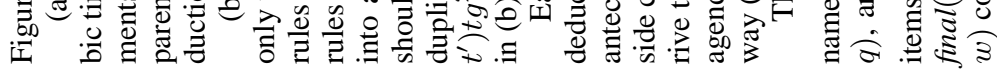

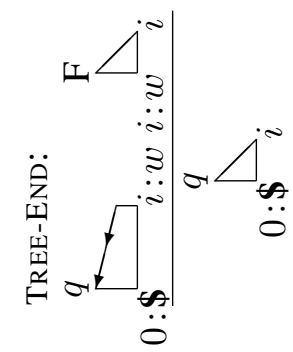

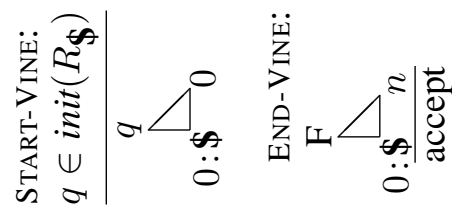

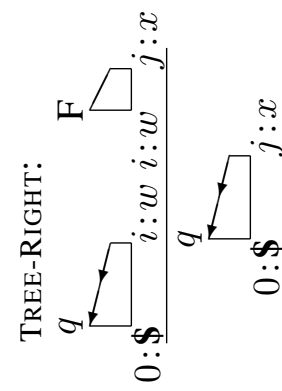

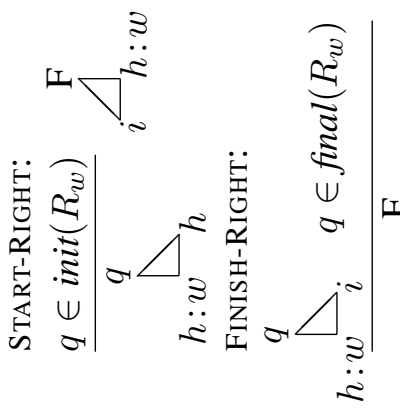
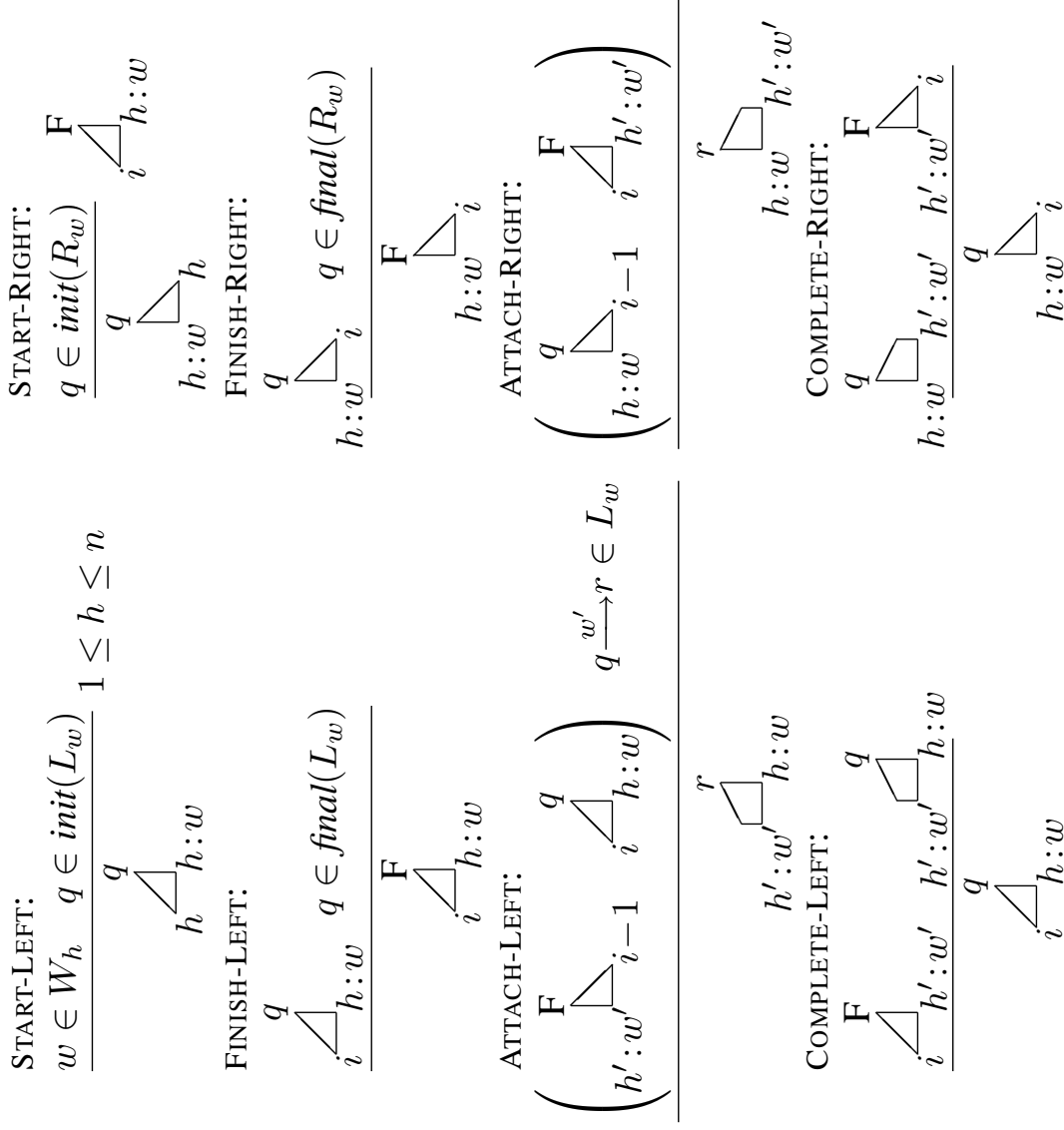

త્త

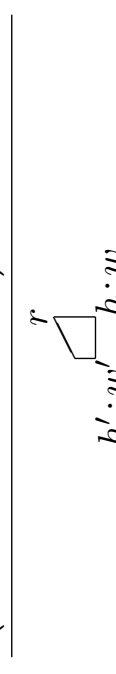




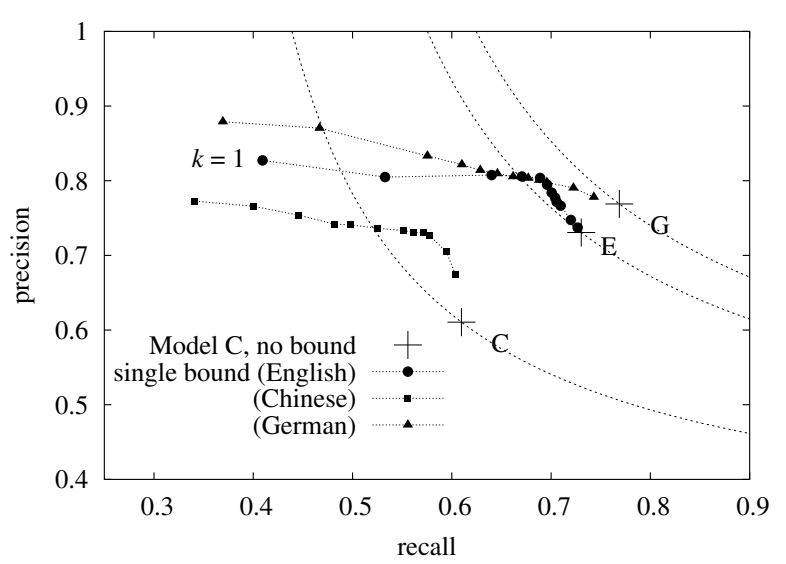

Figure 3: Trading precision and recall: Imposing bounds can improve precision at the expense of recall, for English and Chinese. German performance suffers more. Bounds shown are $k=\{1,2, \ldots, 10,15,20\}$. The dotted lines show constant $F$ measure of the unbounded model.

\subsection{Experiments with Hard Constraints}

Our experiments used the asymptotically fast hybrid parsing algorithm above. We used the same left and right automata as in model $\mathrm{C}$, the best-performing model from $\S 3.2$. However, we now define $R_{\$}$ to be a first-order (bigram) Markov model (§4.1). We trained and tested on the same headed treebanks as before (§3.7), except that we modified the training trees to make them feasible ( $\S 4.2)$.

Results are shown in Figures 3 (precision/recall tradeoff) and 4 (accuracy/speed tradeoff), for $k \in$ $\{1,2, \ldots, 10,15,20\}$. Dots correspond to different values of $k$. On English and Chinese, some values of $k$ actually achieve better $F$-measure accuracy than the unbounded parser, by eliminating errors. ${ }^{23}$

We observed that changing $R_{\$}$ from a bigram to a unigram model significantly hurt performance, showing that it is in fact useful to empirically model likely sequences of parse fragments.

\subsection{Finer-Grained Hard Constraints}

The dependency length bound $k$ need not be a single value. Substantially better accuracy can be retained if each dependency type-each $(h, c, d)=$ (head tag, child tag, direction) tuple-has its own

\footnotetext{
${ }^{23}$ Because our prototype implementation of each kind of parser (baseline, soft constraints, single-bound, and typespecific bounds) is known to suffer from different inefficiencies, runtimes in milliseconds are not comparable across parsers. To give a general idea, 60 -word English sentences parsed in around $300 \mathrm{~ms}$ with no bounds, but at around $200 \mathrm{~ms}$ with either a distance model $p(\Delta \mid d, h, c)$ or a generous hard bound of $k=10$.
}

bound $k(h, c, d)$. We call these type-specific bounds: they create a many-dimensional space of possible parsers. We measured speed and accuracy along a sensible path through this space, gradually tightening the bounds using the following process:

1. Initialize each bound $k(h, c, d)$ to the maximum distance observed in training (or 1 for unseen triples). ${ }^{24}$

2. Greedily choose a bound $k(h, c, d)$ such that, if its value is decremented and trees that violate the new bound are accordingly broken, the fewest dependencies will be broken. ${ }^{25}$

3. Decrement the bound $k(h, c, d)$ and modify the training data to respect the bound by breaking dependencies that violate the bound and "grafting" the loose portion onto the vine. Retrain the parser on the training data.

4. If all bounds are not equal to 1 , go to step 2 .

The performance of every $200^{\text {th }}$ model along the trajectory of this search is plotted in Fig. $4 .{ }^{26}$ The graph shows that type-specific bounds can speed up the parser to a given level with less loss in accuracy.

\section{Related Work}

As discussed in footnote 3, Collins (1997) and McDonald et al. (2005) considered the POS tags intervening between a head and child. These soft constraints were very helpful, perhaps in part because they helped capture the short dependency preference $(\S 2)$. Collins used them as conditioning variables and McDonald et al. as log-linear features, whereas our $\S 3$ predicted them directly in a deficient model.

As for hard constraints ( $(4)$, our limitation on dependency length can be regarded as approximating a context-free language by a subset that is a regular

\footnotetext{
${ }^{24}$ In the case of the German TIGER corpus, which contains non-projective dependencies, we first make the training trees into projective vines by raising all non-projective child nodes to become heads on the vine.

${ }^{25}$ Not counting dependencies that must be broken indirectly in order to maintain projectivity. (If word 4 depends on word 7 which depends on word 2, and the $4 \rightarrow 7$ dependency is broken, making 4 a root, then we must also break the $2 \rightarrow 7$ dependency.)

${ }^{26}$ Note that $k(h, c$, right $)=7$ bounds the width of $\triangle+$ $\Lambda=\square$. For a finer-grained approach, we could instead separately bound the widths of $\$ and $\Delta$, say by $k_{r}(h, c$, right $)=4$ and $k_{l}(h, c$, right $)=2$.
} 
language. Our "vines" then let us concatenate several strings in this subset, which typically yields a superset of the original context-free language. Subset and superset approximations of (weighted) CFLs by (weighted) regular languages, usually by preventing center-embedding, have been widely explored; Nederhof (2000) gives a thorough review. We limit all dependency lengths (not just centerembedding). ${ }^{27}$ Further, we derive weights from a modified treebank rather than by approximating the true weights. And though regular grammar approximations are useful for other purposes, we argue that for parsing it is more efficient to perform the approximation in the parser, not in the grammar.

Brants (1999) described a parser that encoded the grammar as a set of cascaded Markov models. The decoder was applied iteratively, with each iteration transforming the best (or $n$-best) output from the previous one until only the root symbol remained. This is a greedy variant of CFG parsing where the grammar is in Backus-Naur form.

Bertsch and Nederhof (1999) gave a linear-time recognition algorithm for the recognition of the regular closure of deterministic context-free languages. Our result is related; instead of a closure of deterministic CFLs, we deal in a closure of CFLs that are assumed (by the parser) to obey some constraint on trees (like a maximum dependency length).

\section{Future Work}

The simple POS-sequence models we used as an experimental baseline are certainly not among the best parsers available today. They were chosen to illustrate how modeling and exploiting distance in syntax can affect various performance measures. Our approach may be helpful for other kinds of parsers as well. First, we hope that our results will generalize to more expressive grammar formalisms such as lexicalized CFG, CCG, and TAG, and to more expressively weighted grammars, such as log-linear models that can include head-child distance among other rich features. The parsing algorithms we presented also admit inside-outside variants, allowing iterative estimation methods for log-linear models (see, e.g., Miyao and Tsujii, 2002).

\footnotetext{
${ }^{27}$ Of course, this still allows right-branching or leftbranching to unbounded depth.
}
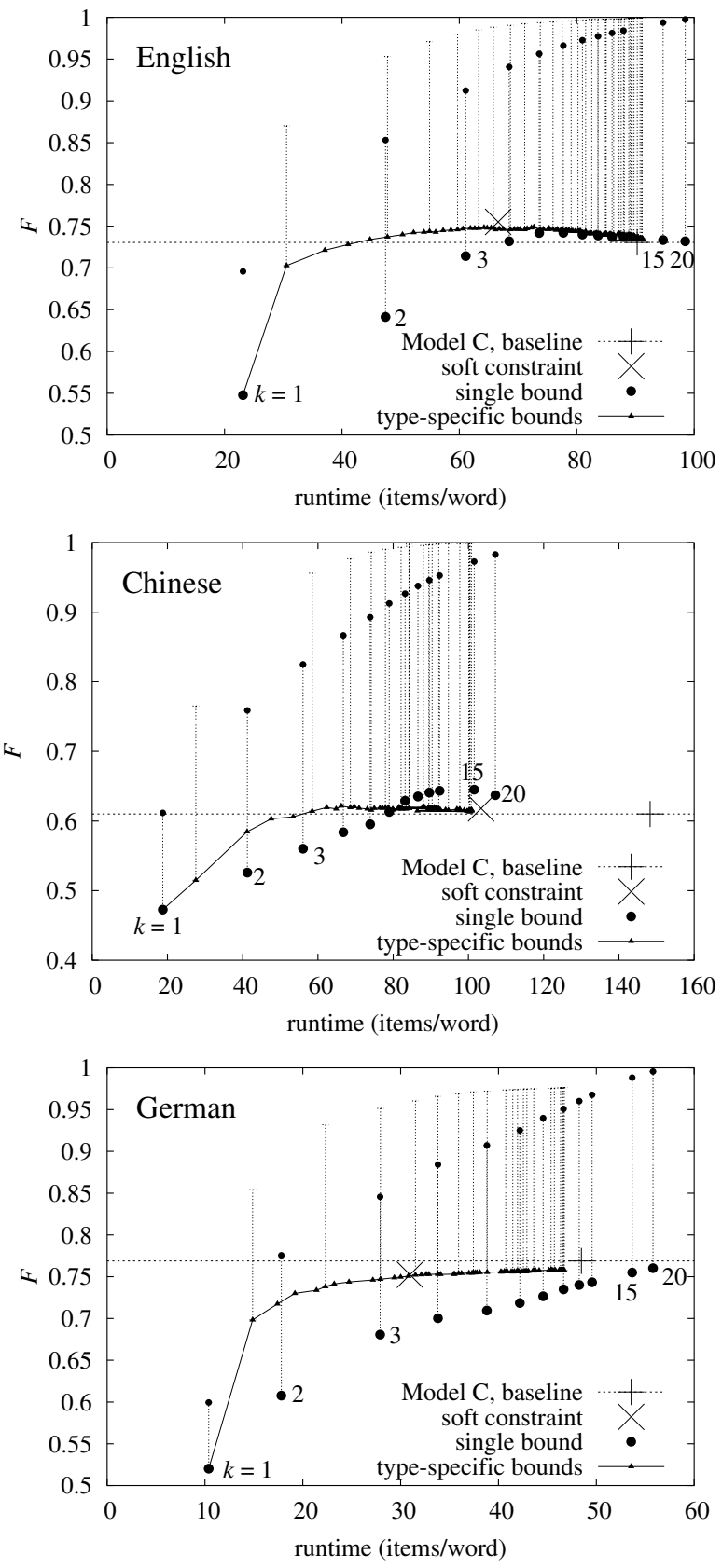

Figure 4: Trading off speed and accuracy by varying the set of feasible parses: The baseline (no length bound) is shown as + . Tighter bounds always improve speed, except for the most lax bounds, for which vine construction overhead incurs a slowdown. Type-specific bounds tend to maintain good $F$ measure at higher speeds than the single-bound approach. The vertical error bars show the "oracle" accuracy for each experiment (i.e., the $F$-measure if we had recovered the best feasible parse, as constructed from the gold-standard parse by grafting: see $\S 4.2)$. Runtime is measured as the number of items per word (i.e., $\triangle, \triangle, \square, \square, \triangle$, $\square$ ) bilt by the agenda parser. The "soft constraint" point marked with $\times$ represents the $p(\Delta \mid d, h, c)$-augmented model from $\S 3$. 
Second, fast approximate parsing may play a role in more accurate parsing. It might be used to rapidly compute approximate outside-probability estimates to prioritize best-first search (e.g., Caraballo and Charniak, 1998). It might also be used to speed up the early iterations of training a weighted parsing model, which for modern training methods tends to require repeated parsing (either for the best parse, as by Taskar et al., 2004, or all parses, as by Miyao and Tsujii, 2002).

Third, it would be useful to investigate algorithmic techniques and empirical benefits for limiting dependency length in more powerful grammar formalisms. Our runtime reduction from $O\left(n^{3}\right) \rightarrow$ $O\left(n k^{2}\right)$ for a length- $k$ bound applies only to a "split" bilexical grammar. ${ }^{28}$ Various kinds of synchronous grammars, in particular, are becoming important in statistical machine translation. Their high runtime complexity might be reduced by limiting monolingual dependency length (for a related idea see Schafer and Yarowsky, 2003).

Finally, consider the possibility of limiting dependency length during grammar induction. We reason that a learner might start with simple structures that focus on local relationships, and gradually relax this restriction to allow more complex models.

\section{Conclusion}

We have described a novel reason for identifying headword-to-headword dependencies while parsing: to consider their length. We have demonstrated that simple bilexical parsers of English, Chinese, and German can exploit a "short-dependency preference." Notably, soft constraints on dependency length can improve both speed and accuracy, and hard constraints allow improved precision and speed with some loss in recall (on English and Chinese, remarkably little loss). Further, for the hard constraint "length $\leq k$," we have given an $O\left(n k^{2}\right)$ partial parsing algorithm for split bilexical grammars; the grammar constant is no worse than for state-ofthe-art $O\left(n^{3}\right)$ algorithms. This algorithm strings together the partial trees' roots along a "vine."

\footnotetext{
${ }^{28}$ The obvious reduction for unsplit head automaton grammars, say, is only $O\left(n^{4}\right) \rightarrow O\left(n^{3} k\right)$, following (Eisner and Satta, 1999). Alternatively, one can convert the unsplit HAG to a split one that preserves the set of feasible (length $\leq k$ ) parses, but then $g$ becomes prohibitively large in the worst case.
}

Our approach might be adapted to richer parsing formalisms, including synchronous ones, and should be helpful as an approximation to full parsing when fast, high-precision recovery of syntactic information is needed.

\section{References}

S. P. Abney. Parsing by chunks. In Principle-Based Parsing: Computation and Psycholinguistics. Kluwer, 1991.

D. E. Appelt, J. R. Hobbs, J. Bear, D. Israel, and M. Tyson. FASTUS: A finite-state processor for information extraction from real-world text. In Proc. of IJCAI, 1993.

E. Bertsch and M.-J. Nederhof. Regular closure of deterministic languages. SIAM J. on Computing, 29(1):81-102, 1999.

D. Bikel. A distributional analysis of a lexicalized statistical parsing model. In Proc. of EMNLP, 2004.

T. Brants. Cascaded Markov models. In Proc. of EACL, 1999.

S. A. Caraballo and E. Charniak. New figures of merit for bestfirst probabilistic chart parsing. Computational Linguistics, 24(2):275-98, 1998.

S. Chen. Bayesian grammar induction for language modeling. In Proc. of ACL, 1995.

$\mathrm{K}$. W. Church. On memory limitations in natural language processing. Master's thesis, MIT, 1980.

M. Collins. Three generative, lexicalised models for statistical parsing. In Proc. of ACL, 1997.

J. Eisner. Bilexical grammars and their cubic-time parsing algorithms. In Advances in Probabilistic and Other Parsing Technologies. Kluwer, 2000.

J. Eisner, E. Goldlust, and N. A. Smith. Compiling Comp Ling: Practical weighted dynamic programming and the Dyna language. In Proc. of HLT-EMNLP, 2005.

J. Eisner and G. Satta. Efficient parsing for bilexical cfgs and head automaton grammars. In Proc. of ACL, 1999.

L. Frazier. On Comprehending Sentences: Syntactic Parsing Strategies. PhD thesis, University of Massachusetts, 1979.

E. Gibson. Linguistic complexity: Locality of syntactic dependencies. Cognition, 68:1-76, 1998.

G. Grefenstette. Light parsing as finite-state filtering. In Proc. of Workshop on Extended FS Models of Language, 1996.

D. Hindle. Noun classification from predicate-argument structure. In Proc. of ACL, 1990.

J. R. Hobbs and J. Bear. Two principles of parse preference. In Proc. of COLING, 1990.

D. Klein and C. D. Manning. Accurate unlexicalized parsing. In Proc. of ACL, 2003.

D. Klein and C. D. Manning. Corpus-based induction of syntactic structure: Models of dependency and constituency. In Proc. of ACL, 2004.

R. McDonald, K. Crammer, and F. Pereira. Online large-margin training of dependency parsers. In Proc. of ACL, 2005.

Y. Miyao and J. Tsujii. Maximum entropy estimation for feature forests. In Proc. of HLT, 2002.

M.-J. Nederhof. Practical experiments with regular approximation of context-free languages. $C L, 26(1): 17-44,2000$.

M.-J. Nederhof. Weighted deductive parsing and Knuth's algorithm. Computational Linguistics, 29(1):135-143, 2003.

C. Schafer and D. Yarowsky. A two-level syntax-based approach to Arabic-English statistical machine translation. In Proc. of Workshop on MT for Semitic Languages, 2003.

B. Taskar, D. Klein, M. Collins, D. Koller, and C. Manning. Max-margin parsing. In Proc. of EMNLP, 2004. 